\title{
A Silicon Magnetic-Field Sensor: Low-Temperature Performance
}

\section{Evaluation}

\author{
P.J. García-Ramírez', F. Sandoval-Ibarra², E.A. Gutiérrez-Domínguez ${ }^{3}$ \\ Centro de Investigación en Micro y Nanotecnología, Universidad Veracruzana, Veracruz (México) \\ Email: jagarcia@uv.mx \\ CINVESTAV, Unidad Guadalajara, Jalisco (México) \\ Email: sandoval@cts-design.com \\ E.A. Gutiérrez-Domínguez, INAOE, Puebla (México)
}

\section{ABSTRACT}

A Si magnetic field-sensitive split-drain MOSFET has been used to study and analyze the effects of a magnetic field on the charge carrier conduction at liquid-nitrogen temperature. In magnetic field sensors (MFS), a key parameter is the Hall angle, which indicates the current line deviation due to the Lorentz force acting on the charge carriers. If temperature is lowered, the carrier mobility increases, therefore, an increase in carrier deflection is expected. To understand the internal deflection of carriers, and optimize the design of a magnetic field sensor, a semi-analytic model has been developed. Using such a model, a MFS has been fabricated and tested. The fi rst experimental results are presented in this work.

KEYWORDS: Transducers, magnetic field sensors, MOS technology, integrated circuits

\section{INTRODUCTION}

Semiconductor Magnetic Field Sensors exploit the galvanomagnetic effects, which are due to the action of the Lorentz force on the charge carriers [1]. In a phosphorous doped semiconductor material, the product of the electron mobility and the magnetic induction causes the sensitivity to magnetic fields [2]. Hence high charge carrier mobility is crucial for achieving high sensitivity. In contrast, let us consider as an additional option the III-V compound semiconductors. They are characterized by high carrier mobility; however, from the cost point of view, silicon is less expensive and offers the unique advantage of MFS compatibility to CMOS technology [3]. Recently, the MFS have experienced a tremendous growth [4]-[6], but the theoretical understanding of the electronic mechanisms underlying these devices has not kept up with this growth. There are several reasons by which the modeling of semiconductor devices in the presence of magnetic fields is required:

- In the bulk, the magnetic field determines a series of effects: changes in the motion of the carrier, the carrier distributions, and the electrostatic potential.

- The magnetic field originates asymmetries into the current equations. In contrast, the boundary conditions retain any zero-field conditions.

Due to the physical structure of a pure crystal, the most fundamental process by which a carrier is scattered is its interaction with the thermally generated vibrations of crystal atoms. At $77 \mathrm{~K}$, for instance, the electrical characteristics of the MFS-based MOSFET improve due to the increase in carrier mobility [7]-[11]. As the magnetic sensitivity is proportional to carrier mobility, the magnetic sensor structure may be able to detect magnetic fields below $1 \mathrm{mT}$ at $77 \mathrm{~K}$ and, by optimizing its design, it would operate with low power consumption $(\mu \mathrm{W})$. 
This paper presents the performance evaluation of a silicon magnetic sensor taken into account the design of a split-drain MOSFET for low-temperature applications. In that sense, basics for the split-drain MOSFET is given in section 2. Experimental results are described in section 3 . Finally, in section 4 the conclusion and future work are presented.

\section{BASICS ON SPLIT-DRAIN MOSFET}

When a magnetic field is applied to a flow of charge carriers (for instance, electrons) moving through a quasi-straight path, they tend to move according to a non-linear trajectory (see Fig. 1) [12]. The deflection of electrons under due to the Lorentz force can be calculated by solving the following equation $\mathrm{B}_{-} \mathrm{B}_{\text {. }}$

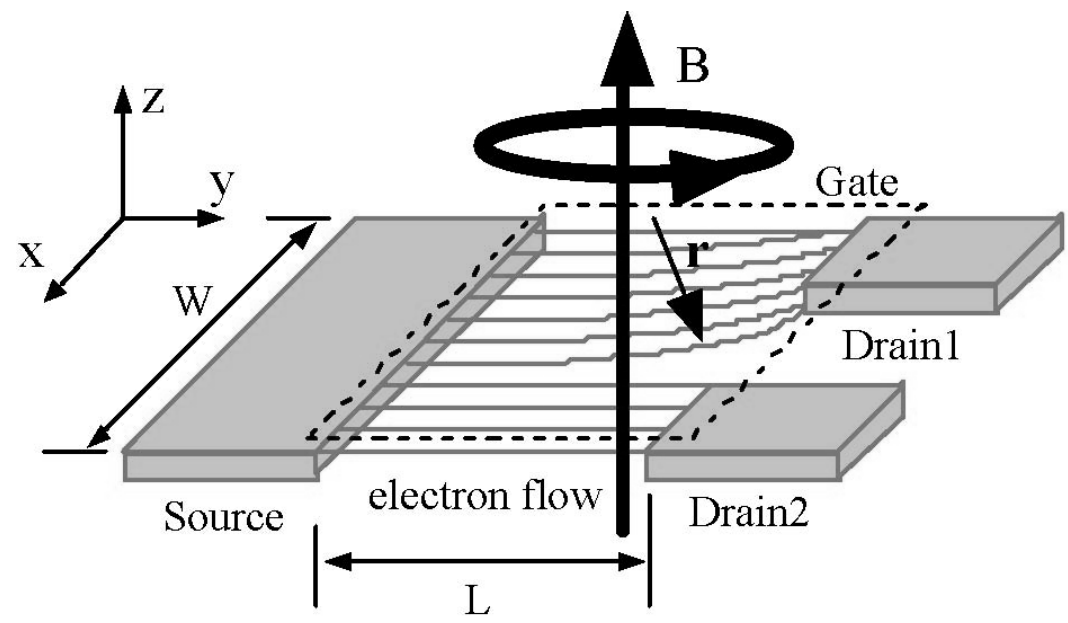

Figure 1. Deflection of electrons under the influence of a magnetic field. Here $\boldsymbol{W}$ and $\mathbf{L}$ are the width and length of the MOSFET inversion layer, respectively. The electron deflection density is measured as a current difference given by $\left(I_{D S 1}{ }_{D S 2}\right)^{\prime}$.

$$
\mathrm{F}_{\mathrm{M}}=\mathrm{q}\left(\mathrm{u}_{\text {drift }} \times \overrightarrow{\mathrm{B}}\right)
$$

As we can see, the higher the drift velocity the higher the magnetic force that translates to a larger deflection. In practice, the distance that electrons deflect from the quasi-straight path is also related to the cyclotron radius

$$
r_{c}=\frac{v_{\text {drift }} m_{n}^{*}}{q|\vec{B}|}
$$

where $\mathbf{m}{ }_{\mathbf{n}}$ is the effective mass of electrons, $\mathbf{q}$ is the magnitude of electronic charge and $v_{\text {drift }}$ is the drift velocity.

It is known that the carrier deflection has been quantified using, as a measurement tool, the Hall effect. This procedure has been used since the end of the XIV century to measure the carrier deflection in extremely thin metallic plates. The last one is commonly named Hall element. In contrast, when the Hall effect is measured in a monolithic Hall element, a low mobility is detected because of the scattering process. On the other hand, the inversion layer of a MOS transistor can be used as a very thin hall element. However, to obtain a uniform structure, the inversion layer must be the channel of a MOSFET 
operating in the linear region $\left(V_{D}<<V_{G}-V_{T}\right)$ [1]. The effective mass of electrons is related to the channel mobility $\boldsymbol{\mu}_{\mathbf{s}}$ as

$$
\mathrm{m}_{\mathrm{n}}^{*}=\frac{\mathrm{q}}{\mathrm{s}} \tau
$$

where $\mathrm{T}$ is the relaxation time. Substituting (3) in (2), we obtain

$$
r_{c}=\frac{v_{\text {drift }} \tau}{\mu_{\mathbf{S}} \vec{B}}=\frac{v_{\text {drift }} \tau}{\tan \Theta_{H}}
$$

The previous relations, where $\Theta_{H}$ is the Hall angle, show that the current line deflection is strongly related to the charge carrier mobility. Hence, the higher the carrier mobility the shorter the curvature radius. For silicon, the charge carrier mobility increases as temperature is lowered; therefore, one expects a larger deflection at cryogenic temperatures. Since the carrier deflection is enhanced at low temperatures, we have designed a Split-Drain MOSFET, where the carrier deflection is measured as a drain current difference $\Delta \mathrm{I}_{\mathrm{DS}}$ (see Fig. 1).

In a MOSFET, when a magnetic induction vector perpendicular is applied to the inversion layer, it produces current imbalance $\Delta=\left(\mathrm{I}_{\mathrm{D} 1}-\mathrm{I}_{\mathrm{D} 2}\right)$ between drains. In this work, the magnetic sensitivity is defined in terms of the relative drain current difference per magnetic induction [13], this is

$$
S_{R}=\left|\frac{\Delta I_{D S}}{I_{D S} \vec{B}}\right|
$$

where $I_{D S}$ is the current supplied by the source region. To describe how the MOSFET works, we propose a semi-analytic model based on semiconductor physics and electromagnetic theory. Some considerations were taken into account [18]-[19]:

- A uniform magnetic-field induction is applied perpendicularly to the gate plane only, and

- the velocity of the ensemble of carriers along the channel is considered to have an average value $v_{\text {drift } t}$

Additionally, the electric fields are labeled $\mathbf{E}_{\mathbf{y}}$ and $\mathbf{E}_{\mathbf{z}}$, where the first/second component is the longitudinal/perpendicular one. To quantify the electric field effect, we propose a model based upon Lorentz Force $\mathbf{F}=q(\mathbf{E}+v$ X B $)$ and Newton's second law. Starting by Lorentz force analysis, we obtain the force components as follows

$$
\begin{gathered}
\mathrm{m} * \frac{\mathrm{d}^{2} \mathrm{x}}{\mathrm{dt}{ }^{2}}=\mathrm{q} \overrightarrow{\mathrm{B}} \mathrm{z} \frac{\mathrm{dy}}{\mathrm{dt}} \\
\mathrm{m} * \frac{\mathrm{d}^{2} \mathrm{y}}{\mathrm{dt}^{2}}=-\mathrm{q} \overrightarrow{\mathrm{B}} \mathrm{z} \frac{\mathrm{dx}}{\mathrm{dt}}+\mathrm{q} \overrightarrow{\mathrm{E}}_{\mathrm{y}} \\
\mathrm{m}^{*} \frac{\mathrm{d}^{2} z}{\mathrm{dt}^{2}}=\mathrm{q} \overrightarrow{\mathrm{E}}_{\mathrm{z}}
\end{gathered}
$$




$$
\begin{aligned}
& \overrightarrow{\mathrm{E}}_{\mathrm{y}}=\frac{\mathrm{V}_{\mathrm{ds}}}{\mathrm{L}}
\end{aligned}
$$

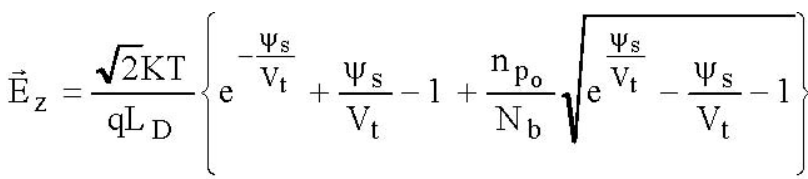

where $\psi_{\mathbf{s}}$ is the surface potential, $\mathbf{V}_{\mathbf{t}}$ the thermal voltage, $\mathbf{k}$ the Boltzmann constant, $\mathbf{L}_{\mathbf{D}}$ the Debye length, $\mathbf{N}_{\mathbf{b}}$ the bulk doping concentration, $\mathbf{n}_{\mathbf{p o}}$ the concentration of electrons in thermal equilibrium in p-type semiconductor, and $\mathbf{T}$ is the absolute temperature. The set of equations (6) can be reduced to

$$
\begin{gathered}
\mathrm{m}^{*} \frac{\mathrm{d}^{2} \mathrm{y}}{\mathrm{dt}^{2}}=-\mathrm{qB}_{\mathrm{z}} \frac{\mathrm{dx}}{\mathrm{dt}}+\mathrm{q} \frac{\mathrm{d}_{\mathrm{ds}} \mathrm{z}}{\mathrm{L}}=\frac{\mathrm{q} \sqrt{2} \mathrm{KT}}{\mathrm{dt} \mathrm{L}_{\mathrm{D}}}\left\{\mathrm{e}^{\left.-\frac{\psi_{\mathrm{s}}}{\mathrm{V}_{\mathrm{t}}}+\frac{\psi_{\mathrm{s}}}{\mathrm{V}_{\mathrm{t}}}-1+\frac{\mathrm{n}_{\mathrm{p}_{\mathrm{o}}}}{\mathrm{N}_{\mathrm{b}}}\left[\mathrm{e}^{\frac{\psi_{\mathrm{s}}}{\mathrm{V}_{\mathrm{t}}}}-\frac{\psi_{\mathrm{s}}}{\mathrm{V}_{\mathrm{t}}}-1\right]\right\}}\right.
\end{gathered}
$$

The trajectories of the carrier are obtained by solving (7) with the inverse Laplace transform method [20][21], with the following initial conditions:

$$
\begin{gathered}
x(0)=0 ;\left.\frac{\partial x}{\partial t}\right|_{t=0}=0 \\
y(0)=0 ;\left.\frac{\partial y}{\partial t}\right|_{t=0}=v_{d r i f t}=\mu_{s} \frac{V_{d s}}{L} \\
z(0)=0 ;\left.\frac{\partial z}{\partial t}\right|_{t=0}=0
\end{gathered}
$$

Once the trajectories of the carrier have been obtained, the following step is to evaluate the time that electrons spend along the channel:

$$
y\left(t_{\text {final }}\right)-L=0
$$

Knowing that time, it is possible to find the carrier deflection under the influence of a magnetic field along the width of the MOSFET.

\section{EXPERIMENTAL RESULTS}

The MFS-based MOSFETs were fabricated on a P-type, (100) 3-in silicon wafers. All Split-Drain MOSFETs were fabricated in the $10 \mu \mathrm{m}$ CMOS MicroElectronics Laboratory of INAOE, at Tonantzintla, Puebla (Mexico). The measured threshold voltage at $300 \mathrm{~K}$ was $\mathrm{V}_{\mathrm{TVN}}=0.96 \mathrm{~V}$. For low-temperature 
testing, the device as well as an electromagnet were immersed in a liquid nitrogen vessel. All data were measured with the HP4156A Semiconductor Parameter Analyzer.

The carrier mobility $\mu$ and magnetic sensitivity $S_{r}$ were estimated from our model and compared to extracted experimental results (see Fig. 2).

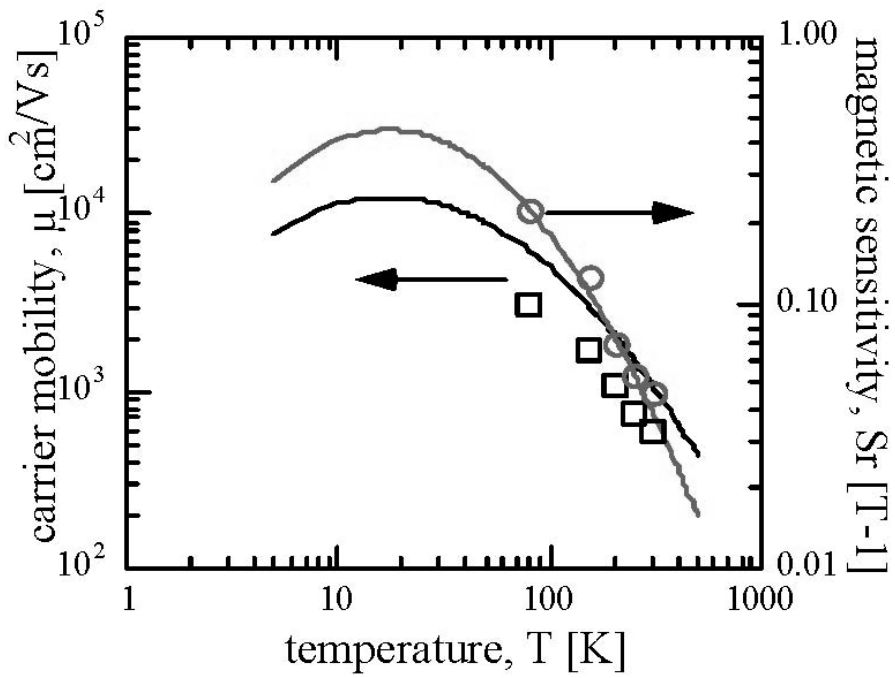

Figure 2. Measured (symbols) and calculated (lines) carrier mobility $\mu$ (squares) and magnetic sensitivity $S_{r}$ (circles).

The increment in the deflection of current lines is confirmed by the augment in carrier mobility and magnetic sensitivity. The deflection of lines $(c d)$ are calculated, and the differential current $\Delta \mathrm{I}_{\mathrm{D}}\left(\left|\mathrm{I}_{\mathrm{D} 1}-\mathrm{I}_{\mathrm{D} 2}\right|\right)$, is also measured at $B=50 \mathrm{mT}$ as a function of temperature (see Fig. 3). As the deflection of current lines increases, due to the increment in carrier mobility, the differential current $\Delta \mathrm{I}_{\mathrm{D}}$ does too.

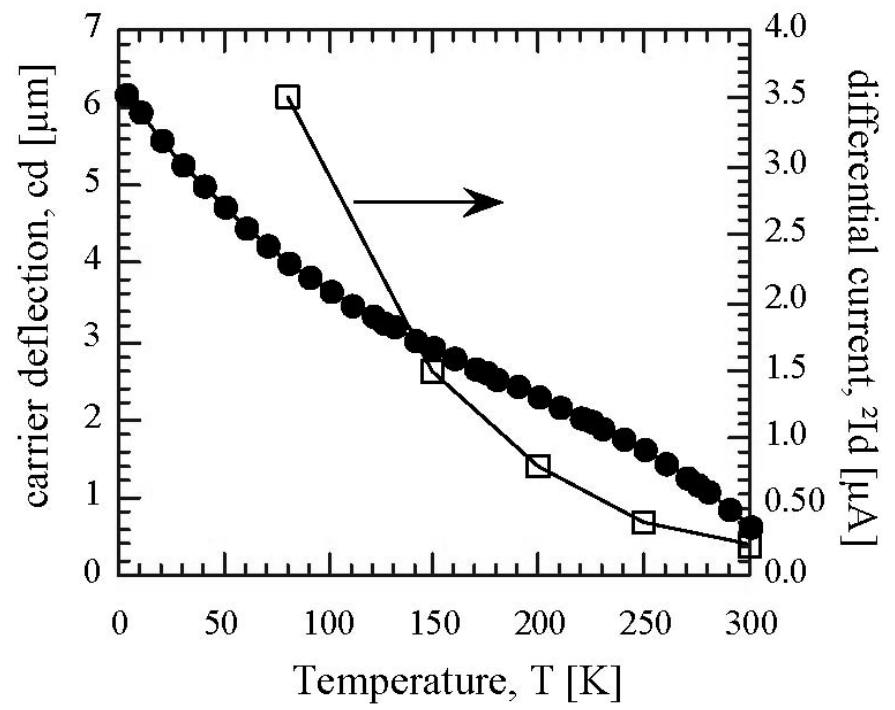

Figure 3. Calculated carrier deflection cd and measured differential current $\Delta / d$ at $B=50 \mathrm{mT}$. 
The differential current, which is result of the carrier mobility and the intensity of the magnetic field, is linearly dependent on the magnetic field itself. This is experimentally shown in Fig. 4. From the latest result, we also confirm the augment in magnetic sensitivity as temperature is lowered. Finally, the power consumption of this magnetic sensor is of about $(5 \mathrm{~V})(30 \mu \mathrm{A})=150 \mu \mathrm{W}$ at $77 \mathrm{~K}$ with a relative sensitivity $\mathrm{S}_{\mathrm{r}}=0.23 \mathrm{~T}^{-1}$, while at room temperature the power consumption is about $110110 \mu \mathrm{W}$ and $\mathrm{S}_{\mathrm{r}}=0.045 \mathrm{~T}^{-1}$. The magnetic sensitivity increases by a factor of 5.1 times when cooled down from room temperature to $77 \mathrm{~K}$. This implies that the minimum magnetic field $\mathrm{B}_{\min }$ that can be sensed could be reduced down to the range of microTeslas $(\mu \mathrm{T})$.

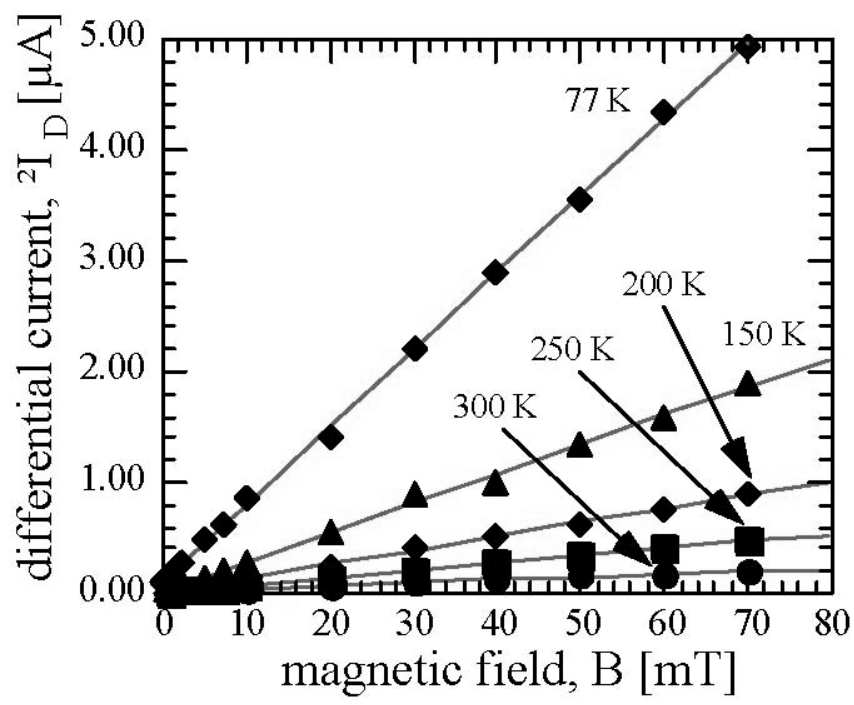

Figure 4. Measured $\Delta I_{D}$ current versus $B$ for different temperatures. In this research the size of the split-drain transistor was $W / L=100 \mu m / 400 \mu m$.

\section{CONCLUSIONS}

To study carrier deflection in Si under the influence of a magnetic field, a semi-analytical model, based on semiconductor physics has been developed. The model has been implemented with the MAPLE software under a PC-based platform. The program allows us to solve the mentioned coupled equation system and give us a quick estimation on the carrier deflection. As a consequence, the carrier deflection is used to determine how many current lines deviate from one drain electrode to the other one. From this estimation, the differential current as well as the magnetic sensitivity can be calculated. This estimation, as a first approximation, is very useful to optimize the design of the MFS-based MOSFET.

\section{REFERENCES} Semiconductors, Adam Hilger, 1990 
[3] Henry P. Baltes \& R. S. Popovic, Proc. of the IEEE, 74 (1986)

[4] V. Zieren and B. P. M. Duyndam, IEEE Trans. Electron Devices, ED-29 (1982) 83

[5] A. W. Vinal and N. A. Masnari, IEEE Electron Device Letters, EDL-3 (1982) 203

[6] R. S. Popovic' and H. P. Baltes, IEEE Electron Device Letters, EDL-4 (1983) 51

[7] P. J. García Ramírez, R.S. Murphy-Arteaga \& E. A. Gutiérrez-Domínguez, Workshop Iberchip, Lima, (1999) 247

[8] Robert M. Fox \& Richard C. Jaeger, IEEE Transactions on electron Devices, ED-34 (1987) 114

[9] Albert K. Hemming, Nelson N. Chan, Jeffrey T. Watt \& James D. Plummer, IEEE Transactions on Electron Devices, ED-34 (1987) 64

[10] Kazuya Masu, You-Wen Yi, Kazuo Tsubouch \& Nobuo Mikoshiba,, Workshop on Low Temperature Semiconductor Electronics, Burlington, (1989) 104

[11] S. F. Voges \& M. Du Plessis,, Workshop on Low Temperature Semiconductor Electronics, Burlington, (1989) 38

[14] Arokia Nathan, Andre M. J. Huiser \& Henry P. Baltes, IEEE Transaction on Electron Devices, ED-32 (1985) 1212

[15] A. Nathan, L. Andor, H. P. Baltes \& H. G. Schmidt-Weinmar, IEEE Journal of Solid-State Circuits, pp 819-821, SC-20 (1985) 819

[16] A. Nathan, W. Allegretto, H. P. Baltes \& Y. Sugiyama, IEEE Transaction on Electron Devices, ED-34 (1987) 2077

[17] B. Ancelle, E. Gallegher \& P. Masse, IEEE Transaction on Magnetics, MAG-18 (1982) 655

[18] Allen Nussbaum \& Ravi Sinha, Solid-State Electronics, 27 (1984) 97

[19] R. F. Pierret \& J. A. Shields, Solid-State Electronics, 26 (1983) 143

[20] Dennis G. Zill, A First Course in Differential Equations with Applications, PWS Publishers, 1988

[21] M. L. Abell \& J. P. Braselton, DIFFERENTIAL EQUATIONS with MAPLE V, AP Professional, 1994 
Authors Biography

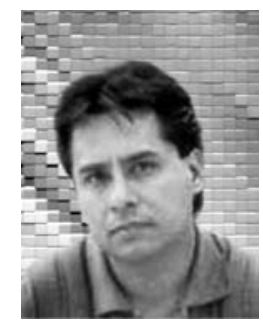

Federico Sandoval-Ibarra

Was born in San Luis Potosí, Mexico. He received the B.E. degree in physics-electronics from the USLP in 1988, Mexico, and the D. Sc. degree in electronics from INAOE, Mexico, in 1997. From 1991 to 1996, he worked in the Microelectronics Laboratory at INAOE as a researcher developing wet-etching techniques and designing CMOS circuitry for silicon-based microsensors. In 1997, he was at CNM, in Bellaterra (Spain), as a visiting researcher being involved in the development of surface micromachining techniques to design a fully-integrated microphone. In 1999, he joined CINVESTAV, Guadalajara Unit, Mexico. From 2002 to 2006, he was the coordinator of the Electronic Design Group. His research areas include silicon-based sensors development, low-voltage low-power circuits design, silicon-based DC-DC converters and mixed-mode circuits for both RFID applications and multi-standard communications.

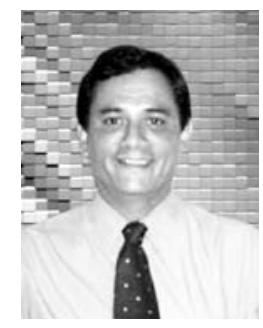

Edmundo A. Gutiérrez-Domínguez

Has published more than 80 papers and international conferences in the field of semiconductor device physics, modeling, simulation, sensors, and circuits. He is author of the book "Low Temperature Electronics, Physics, Devices, Circuits and Applications" (Academic Press, 2000). Prof. Gutierrez has supervised $4 \mathrm{M}$.Sc. and $10 \mathrm{PhD}$ theses. He has been invited Professor at Simon Fraser University (Vancouver, Canada), University of Sao Paulo (Brazil), and the Technical University of Vienna, (Austria). From 2000 to 2002, he was the Design Manager of the Motorola Mexico Center for Semiconductor Technology, and from 2005 to 2007, Research Manager of the Intel Mexico Research Center. Currently, Prof. Gutierrez is with the Department of Label1 Electronics of INAOE. Prof. Gutiérrez is member of the National Systems of Researchers (SNI level 2) of Mexico, and member of different Review Committees of the Mexico National Council of Science and Technology (CONACyT). 


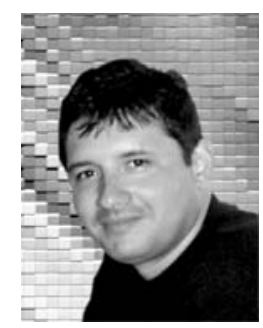

\section{Pedro J. García-Ramírez}

Received the B.S. degree from the Technological Institute of Veracruz, Mexico, in 1993 and the M.Sc. and $\mathrm{Ph}$. D. degree in microelectronics from the National Institute for Astrophysics, Optics and Electronics, in 1997 and 2000, respectively. From 2000 to 2001, he was part of the former On Semiconductor Technical Operations Group in Jalisco, Mexico as a Senior Development Engineer. He joined the faculty of Universidad Veracruzana, in Boca del Rio, Mexico in 2001, where he currently holds the rank of Fulltime Professor in the Electronics and Communications Department and, since August 2005, he has been director of the Research Center for Micro and Nanotechnology (MICRONA). His research interests span from CMOS compatible magnetic sensors modeling to characterization techniques. 